Original Article

\title{
MUTANT P21 PEPTIDES COULD ACT AS AN IMPROVED CYCLIN A INHIBITORS FOR CANCER THERAPY: AN IN SILICO VALIDATION
}

\author{
TARUN AGARWAL ${ }^{1}$, NITHYANAN ANNAMALAI ${ }^{2}$, HARI PRASAD RONANKI ${ }^{1}$, SANDHYA BUTTY ${ }^{1}$, TAPAS KUMAR \\ MAITI $^{*}$, HASNI BIN ARSAD ${ }^{2}$
}

${ }^{1}$ Department of Biotechnology, Indian Institute of Technology Kharagpur, Kharagpur, West Bengal 721302, India, ${ }^{2}$ Advanced Medical and Dental Institute, Universiti Sains Malaysia, 13200, Bertam, Pulau Pinang, Malaysia

Email: tkmaiti@hijli. iitkgp.ernet.in

Received: 31 Oct 2018 Revised and Accepted: 20 Dec 2018

\section{ABSTRACT}

Objective: The present study delineates the generation of mutant peptide library from a known anticancer peptide, p21 and in silico evaluation for their affinity towards cyclin. A substrate binding groove.

Methods: Mutant peptide library was created based on their AntiCP score and was docked with cyclin A using ClusPro2.0 web server. The docked structures were further simulated into an aqueous environment using Gromacs 4.5.6. Visualization was performed using PyMol software and interaction analysis was done using Discovery Studio Visualizer 4.1 Client and LigPlot plus tool.

Results: A total of 57 mutant peptides were generated; out of which only 3 namely, K3C (Lys3Cys), K3F (Lys3Phe), and K3W (Lys3Trp) had a greater affinity for cyclin A than WILD p21 peptide (HSKRRLIFS). Molecular dynamic simulation studies showed that the peptides remained docked into the substrate binding groove throughout the run. Among all the peptides, K3C showed a significantly higher negative binding energy with cyclin A as compared to WILD.

Conclusion: The overall results suggested that K3C mutant peptide had $\sim 30 \%$ higher affinity towards cyclin A and thus, could further be explored for its anticancer potential. The study also provides an insight into the crucial interactions governing the recognition of substrate binding groove of cyclin A for the development of novel peptide-based anticancer therapeutics.

Keywords: Target-based cancer therapy, Cyclin A, p21 mutant peptide library, Molecular docking, Molecular dynamic simulation

(c) 2019 The Authors. Published by Innovare Academic Sciences Pvt Ltd. This is an open access article under the CC BY license (http://creativecommons.org/licenses/by/4.0/) DOI: http://dx.doi.org/10.22159/ijpps.2019v11i2.30577

\section{INTRODUCTION}

In the past few decades, cancer has emerged as a leading cause of death worldwide. Annually, cancer causes an estimated death of 5.5 lakh people and $\sim 8$ lakh new cases are at its risk [1,2]. Conventional therapies including chemo-and radio-therapies have been associated with a series of side-effects [2]. In addition to cancer cells, these therapies are also detrimental to the healthy cells and further result in many other systemic side-effects. Lack of selectivity of these therapies has made researchers focus on the development of targetbased strategies for cancer treatment [3].

Under normal conditions, DNA damage activates tumor suppressor cascade resulting in either repair or growth arrest or apoptosis of the damaged cell $[4,5]$. DNA damage is generally associated with the activation of p53 which in-turn activates a series of other proteins including DNA repair proteins, cell proliferation blockers and apoptosis-inducing proteins [6, 7]. On the other hand, cancerous conditions are associated with the aberrant version of either of these transcription factors; making the cell incapable to sense DNA damage and/or any deleterious mutation, thus, leading to an uncontrolled growth and transformation of the damaged cells.

The interactions between the biological molecules such as proteinprotein, protein-DNA, and protein-RNA are considered to be very specific. Therefore, small drug molecules (both protein and non-protein based) and antibody-based therapeutic molecules such as Gleevec, Bombesin, Octreotide, Avastin, Herceptin, Iressa, Erbutix, etc., have come up in the commercial market for more effective cancer treatment $[2,3]$. Moreover, small peptide-based drugs have also presented a suitable alternative for cancer treatment. The peptides, being smaller in size, could easily be internalized by the cells, could specifically bind to their target and could either activate or block the targeted signaling cascade $[8,9]$. Such potency of the peptides has offered a new area for exploration of novel peptide-based therapeutics. Present day research focuses on identifying bioactive peptide from proteins that are known to interact with oncogenic proteins and thereby specifically targeting only the cancerous cells.

In this regard, peptides derived from tumor suppressor proteins such as p53, p27, p21, pRB, p107, p130, etc. have proved to be of significant importance [10-12]. These proteins have long been known to exert tumor suppression by interacting with CDK/cyclin complex. Peptides derived from these proteins contain a minimal recognition motif which could specifically interact with the substrate binding groove on cyclin, thereby preventing recruitment and phosphorylation of CDK substrates and thus, blocking the cell cycle progression [10].

Previously, Zheleva et al. has demonstrated the anticancer potential of the p21 C-terminal peptide "HSKRRLIFS" [10]. But there still exists an urge to develop a more efficient therapeutics to combat cancer and its increasing resistance towards the current therapeutics. In this regard, a better strategy would be to modify the pre-existing anti-cancer agents instead of investing in the development of new therapeutic from scratch. Keeping the aforesaid perspective in mind, herein, we present in silico evaluation of p21 based mutant peptides for their anticancer potential. Here, we have applied a molecular docking and molecular dynamic simulation-based strategies for screening purpose.

\section{MATERIALS AND METHODS}

Cyclin A structure retrieval and prediction of the peptide docking site

X-ray crystallographic structures of human cyclin A and p21 peptide were retrieved from Protein Data Bank (PDB) with Ids-1JSU [13] and 1AXC [14] respectively. Both the structures were cleaned using Argus Lab software. For peptide, a specific sequence "HSKRRLIFS" was isolated for the creating the mutant peptide library and further molecular docking evaluations. Substrate binding groove of cyclin A 
was analyzed by evaluating its interaction with p27 peptide in 1JSU PDB file using Discovery Studio Visualizer 4.1 Client.

\section{Preparation of p21 mutant peptide library and molecular} docking with cyclin A

The initial selection of the mutant peptide was carried out based on their higher AntiCP score in comparison to the WILD peptide (AntiCP score $=$ 0.70) generated using AntiCP: Prediction and Designing of Anticancer Peptides web server (http://crdd. osdd. net/raghava/anticp/) [15]. Selected mutant peptides were generated using mutagenesis module of the PyMOL software (DeLano Scientific). Further, docking of the mutant peptide library was carried out using the ClusPro 2.0 web server (https://cluspro. bu. edu/home. php) in an automated mode [16, 17]. Mean and lowest energy of the complex along with their interaction profile formed a basis of peptide screening for molecular dynamic simulation-based validation. Interaction profile of the peptides was analyzed by Discovery Studio Visualizer 4.1 Client.

\section{Molecular dynamic simulation-based evaluation of cyclin A-} peptide complexes

Molecular dynamic simulation of screened cyclin A-peptide complex was carried out in an aqueous environment with a salt concentration of $0.156 \mathrm{M}$ under GROMOS96 53a6 force field using Gromacs 4.5.6 package following the protocol previously reported elsewhere [1]. The simulation was carried out for 20 ns timescale. Analysis of protein-peptide binding energy during the simulation run was done using MM/PBSA analysis. The work was carried out at the computational facility present at Advanced Medical and Dental Institute, Universiti Sains Malaysia, Malaysia. Visualization of the complexes was carried out using PyMOL software (DeLano Scientific) and their interaction profiling was examined by Discovery Studio Visualizer 4.1 Client and LigPlot plus tool.

\section{RESULTS AND DISCUSSION}

\section{Evaluation of cyclin A substrate binding site}

In the previous reports, Zheleva et al. have already demonstrated that the peptides derived from the C-terminus of p21 could effectively interact with cyclin A through its cyclin groove recognition sequence "SKRRLIF" [10]. Such an interaction prevents binding and phosphorylation of downstream proteins by CDK2/cyclin A complex, thereby, blocking cell cycle progression. Moreover, a similar cyclin-groove recognition sequence "ACRNLFG" was also present in the p27 peptide structure of the ternary complex of CDK2/Cyclin A/p27 (accession number-1JSU). Thus, it was used to first analyze the substrate binding groove of cyclin $\mathrm{A}$. The results demonstrated that the substrate binding groove was formed by $\alpha 1$, $\alpha 3$ and $\alpha 4$ helixes in the N-terminal domain of cyclin A. Amino acid residues Met210, Ile213, Trp217, Glu220, Val221, Arg250, Gln254, Tyr280, Ile281, Thr282, and Thr285 of cyclin A formed a crucial part of the docking site. Arg5 of p27 peptide extended towards $\alpha 1$ helix forming hydrogen bonds with Glu220 and Ile281. Leu7 and Phe8 of p27 were found buried into the hydrophobic pocket formed by Met210, Ile213, and Trp217, thereby extending stabilizing to the protein-peptide complex.

Molecular docking of the WILD p21 peptide was done using ClusPro 2.0 web server. Previous studies have efficiently exploited the server for studying protein-peptide interactions [18, 19]. The results revealed that WILD peptide docked into the substrate binding groove in a similar orientation as that of the p27 peptide with a ClusPro weighted docking score of-805.6. Further, the simulationbased evaluations of the complex demonstrated WILD peptide to be docked into the substrate binding groove throughout the simulation run (fig. 1). From the energy decomposition analysis, electrostatic energy was found to have a greater contribution to the overall binding energy of the peptide. Per-residue energy decomposition study further revealed that amino acid residues Met210, Ile213, Asp216, Trp217, Glu220, Glu223, Glu224, Arg250, Gln254, Tyr280, Ile281, Asp283, and Asp284 of cyclin A were important for its interaction with WILD peptide (fig. $1 \mathrm{c}$ ). From the analysis, it was very evident that the cationic amino acid residues of p21 (Lys3, Arg4, and Arg5) interacted with anionic residues of cyclin A (Asp216, Glu220, Glu223, Glu224, Asp283, and Asp284); while hydrophobic residues, Leu6, and Phe8 of p21 interacted with Trp217, Ile213, Leu214, Leu253, and Ile281 of cyclin A.

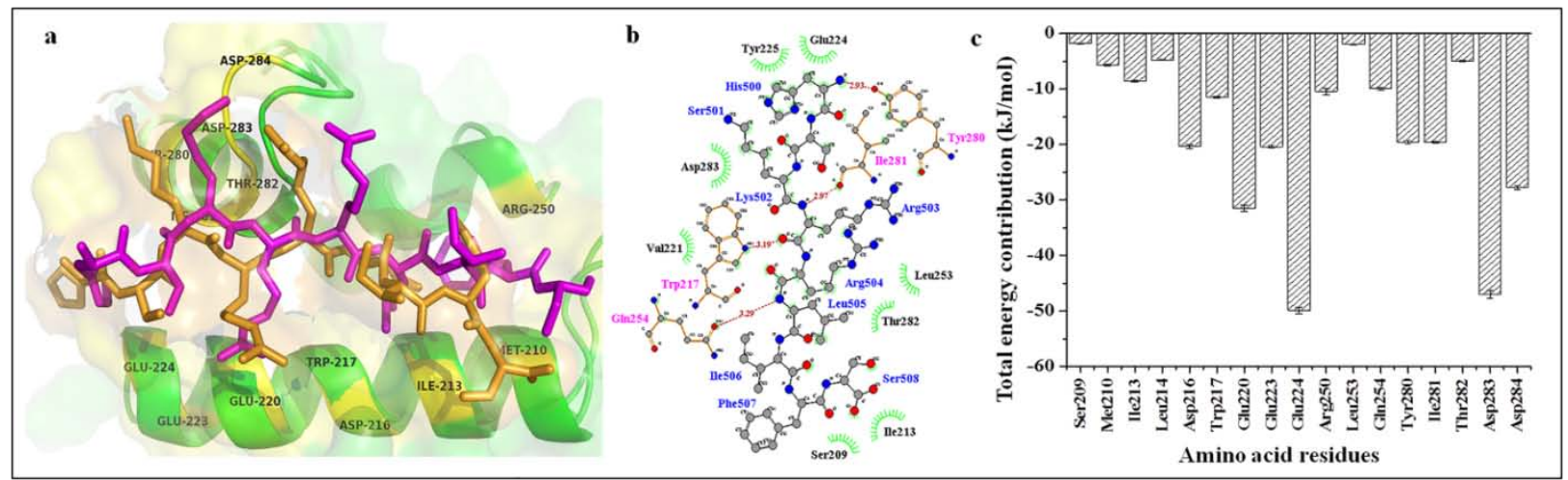

Fig. 1: Docking and simulation analysis of WILD p21 peptide. (a) The binding pose of the p21 peptide after docking (magenta) and molecular dynamic simulation (orange). Interacting amino acid residues have been highlighted in yellow. (b) LigPlot plus based interaction analysis of cyclin A/WILD peptide complex. (c) Per-residue energy decomposition analysis of cyclin A/WILD peptide complex. Amino acid His500-Ser508 of WILD/K3C peptide in the LigPlot plus 2D representation corresponds to His1-Ser9 (in reference to other sections of the manuscript)

\section{Generation of mutant peptide library and docking with cyclin A}

Previously, Zheleva et al., through an in vitro alanine mutagenesis analysis has demonstrated that amino acid residues Arg4, Leu6, and Phe8 of WILD peptide were crucial for effective binding with cyclin A [10]. In order to validate our model with these experimental results, we first generated a series of alanine mutants of the WILD p21 peptide including H1A, S2A, K3A, R4A, R5A, L6A, I7A, F8A, and S9A, followed by docking and molecular dynamic simulation analysis (Refer supplementary section S1). The results obtained suggested that our working model was consistent with the previously reported experimental results with R4A, L6A and F8A showing a maximum reduction in their binding affinity and loss of interaction with cyclin A (refer Supplementary table S1-S4 and fig. S1).

With this analogy, we created mutant p21 peptide library keeping amino acid residues Arg4, Leu6, and Phe8 unaltered. Here, we further employed AntiCP server to screen and select the efficient peptides based on their higher SVM score in comparison to WILD peptide (SVM score wILD $=0.70$ ). AntiCP is a web server which applies SVM model based on amino acid composition and binary profile features to predict the potential of the peptide as an anticancer agent [15]. During initial screening, a total of 57 mutant peptide sequences with a score $>=0.70$ were obtained. Thereafter, three-dimensional peptide structure was generated using 
mutagenesis module of PyMOL software followed by docking with cyclin A (refer supplementary table S5-S10).

The docking results demonstrated that only 11 out of predicted 57 peptides; had ClusPro weighted docking score higher than WILD including S2R, K3C, K3F, K3P, K3W, K3Y, I7C, I7M, S9C, and S9Q mutants (table 1). A closer analysis demonstrated that most of the peptides interacted with crucial amino acid residues of cyclin $\mathrm{A}$ including Asp216, Trp217, Glu220, Glu223, Glu224, Arg250, Gln254, Tyr280, Ile281, Asp283, and Asp284 (table 2).

Table 1: Cyclin A-selected random mutant's binding energy

\begin{tabular}{lll}
\hline Peptides & Lowest energy & Normalized lowest energy \\
\hline Wild & -805.6 & 1 \\
I7C & -812.5 & 1.0086 \\
I7M & -806.5 & 1.0011 \\
K3C & -857 & 1.0638 \\
K3F & -860.9 & 1.0686 \\
K3P & -815.8 & 1.0127 \\
K3W & -847.7 & 1.0523 \\
K3Y & -825.6 & 1.0248 \\
S2R & -838.8 & 1.0412 \\
S9C & -823.9 & 1.022 \\
S9Q & -829.4 & 1.0295 \\
\hline
\end{tabular}

Number of experiments, $\mathrm{n}=1$.

Table 2: Cyclin A-selected random mutant's interaction analysis

\begin{tabular}{ll}
\hline Mutants & Interacting amino acids \\
\hline I7C & Ile213, Trp217, Glu220, Glu224, Arg250, Leu253, Gln254, Tyr280, Ile281, Asp283, Asp284, Thr285 \\
I7M & Trp217, Glu220, Glu224, Arg250, Leu253, Gln254, Tyr280, Ile281, Asp283, Asp284, Thr285 \\
K3C & Ile213, Leu214, Asp216, Trp217, Glu220, Glu224, Arg250, Leu253, Gln254, Tyr280, Ile281, Asp283, Thr285 \\
K3F & Asp216, Trp217, Glu220, Gln254, Tyr280, Ile281, Asp283, Asp284, Thr285, Ser408 \\
K3P & Asp216, Trp217, Glu220, Glu224, Arg250, Leu253, Gln254, Tyr280, Asp283, Thr285, Ser408, \\
K3W & Asp216, Trp217, Glu220, Arg250, Gln254, ,p283, Thr285, Ser408, Lys412 \\
K3Y & Asp216, Trp217, Glu220, Glu224, Gly251, Gln254, Tyr280, Ile281, Asp283, Asp284, Tyr286, Ser408, Glu411 \\
S2R & Met210, Asp216, Trp217, Glu220, Glu224, Arg250, Leu253, Gln254, Ile281, Thr285, Tyr280, Asp83, Asp284 \\
S9C & Met210, Ile213, Leu214, Asp216, Trp217, Glu220, Glu224, Arg250, Gln254, Ile281 \\
S9Q & Ile213, Asp216, Trp217, Glu220, Glu224, Arg250, Leu253, Gln254, Tyr280, Asp283, Asp284, Thr285, Ser408 \\
\hline
\end{tabular}

In the present study, we selected peptides with at least $5 \%$ higher ClusPro weighted docking score with a binding orientation similar to that of WILD peptide which includes K3C, K3F, and K3W. Weighted docking score for the $\mathrm{K} 3 \mathrm{C}, \mathrm{K} 3 \mathrm{~F}$, and $\mathrm{K} 3 \mathrm{~W}$ mutant peptides was-857.0,860.9, and-847.7 which was higher than that of WILD (-805.6). A close insight into the binding pattern of the Lys3 mutant peptides showed distinct variations in comparison to the WILD. Substitution of lysine with bulky hydrophobic residues (either phenylalanine or tryptophan as in $\mathrm{K} 3 \mathrm{~F}$ and $\mathrm{K} 3 \mathrm{~W}$ mutants respectively); completely altered the binding orientation of the peptides. Both K3F and K3W peptides rotated by about an angle of $30^{\circ}$ with respect to the WILD peptide, extending over $\alpha 1$ helix instead of docking into substrate binding groove. His1 residues of $\mathrm{K} 3 \mathrm{~F}$ and $\mathrm{K} 3 \mathrm{~W}$ interacted with $\alpha$-C terminal helix which was absent in the WILD. The bulky amino acid residues, Phe3, and Trp3 in K3F and K3W respectively; docked into the hydrophobic pocket formed by Trp217, Val221, and Ile281. In contrast, peptide $\mathrm{K} 3 \mathrm{C}$ docked into the substrate binding groove of cyclin A. Substitution of lysine with cysteine residue in K3C peptide resulted in slightly greater folding of the peptide which slightly affected its interaction pattern with cyclin A. Cys3 residue in $\mathrm{K} 3 \mathrm{C}$ peptide interacted with Tyr280 of cyclin A. Arg4 in K3C interacted with Asp216, Glu220, Gln254, Ile281, Leu270, and Trp217; while it interacted with Glu220, Glu224, Gln254, Ile281, Thr282, and Trp217 in WILD. Arg5 formed hydrogen bond interactions with Asp283 and Thr285; while Leu6 interacted with Trp217 and Gln254. These interactions were common to both WILD and K3C peptides. In K3C, Phe8 established interactions with Arg250, Leu253, Ile213, and Leu214 in comparison to Leu253 only in WILD, which may support a higher binding affinity of K3C than WILD peptide. Further validation of these results was carried out using a molecular dynamics simulation of the cyclin A-WILD/K3C complexes.

\section{Molecular dynamic simulation and MM/PBSA analysis of cyclin} A/peptide complexes

Based on the initial docking and interaction profiling, both cyclin AWILD/K3C complexes were evaluated for stability of their interaction in an aqueous environment using molecular dynamics simulation. The study revealed that cyclin $\mathrm{A} / \mathrm{K} 3 \mathrm{C}$ complex had a higher flexibility in comparison to cyclin A/WILD complex. Root mean square deviation (RMSD) of cyclin A protein showed higher fluctuations in K3C (fig. 2 a). RMSD values of cyclin A in complex with WILD and K3C peptides were 0.367 and $0.375 \mathrm{~nm}$ respectively at the end of the analysis of 20 ns. Root mean square fluctuation (RMSF) plot demonstrated that in $\mathrm{K} 3 \mathrm{C}$, the zone of higher deviation was mainly restricted to the substrate binding groove and Cterminal of cyclin A (fig. $2 \mathrm{~b}$ ). All the substrate binding site residues namely, Ser209, Met210, Ile213, Leu214, Asp216, Trp217, Glu220, Glu224, Leu253, Gln254, Tyr280, Ile281, Thr282, Asp283, Asp284 except Glu223, and Arg250 exhibited higher fluctuation in cyclin $\mathrm{A} / \mathrm{K} 3 \mathrm{C}$ complex. At gross, these residues exhibited a greater fluctuation in their RMSD profiles in cyclin $\mathrm{A} / \mathrm{K} 3 \mathrm{C}$ complex in comparison to cyclin A/WILD complex throughout the simulation run (fig. 2 c). Such fluctuations in the RMSD profile suggest a constant rearrangement of the $\mathrm{K} 3 \mathrm{C}$ peptide and corresponding cyclin A interacting residues to achieve a stable conformation. Hydrogen bond plot demonstrated that $\mathrm{K} 3 \mathrm{C}$ peptide formed a higher number of bonds in comparison to the WILD peptide which supports its greater affinity for cyclin A as concluded from docking studies (fig. 2 d). The average number of hydrogen bonds formed by WILD and $\mathrm{K} 3 \mathrm{C}$ were $6.895 \pm 1.875$ and $9.995 \pm 2.216$ respectively. All these results clearly indicate molecular and structural rearrangements induced by $\mathrm{K} 3 \mathrm{C}$ in the complex.

From the trajectory analysis, it was evident that both WILD and K3C mutant peptides remained docked into the substrate binding groove of cyclin A throughout the simulation run. During this period, Cterminal of WILD peptide showed adjustment of Leu6 and Phe8 into the hydrophobic pocket. Phe8 of WILD peptide moved slightly outward from the hydrophobic groove thus, losing its interaction with Leu214 and Leu253 at the end of 20 ns. In K3C, the N-terminal of peptide rolled into the substrate binding groove. This was further validated by a decrease in radius of gyration $(\mathrm{Rg})$ and solvation area 
(SASA) of the K3C peptide with time (fig. 2 e and f). Such a folding of K3C peptide allowed His1 to form interactions with Glu220 and Glu224 which were missing from the initial docked structure.
Moreover, Ser2 formed additional hydrogen bonds with Trp217 and Glu224. Formation of these additional interactions further supported the initial docking results (table 3, table 4 and fig. 3).

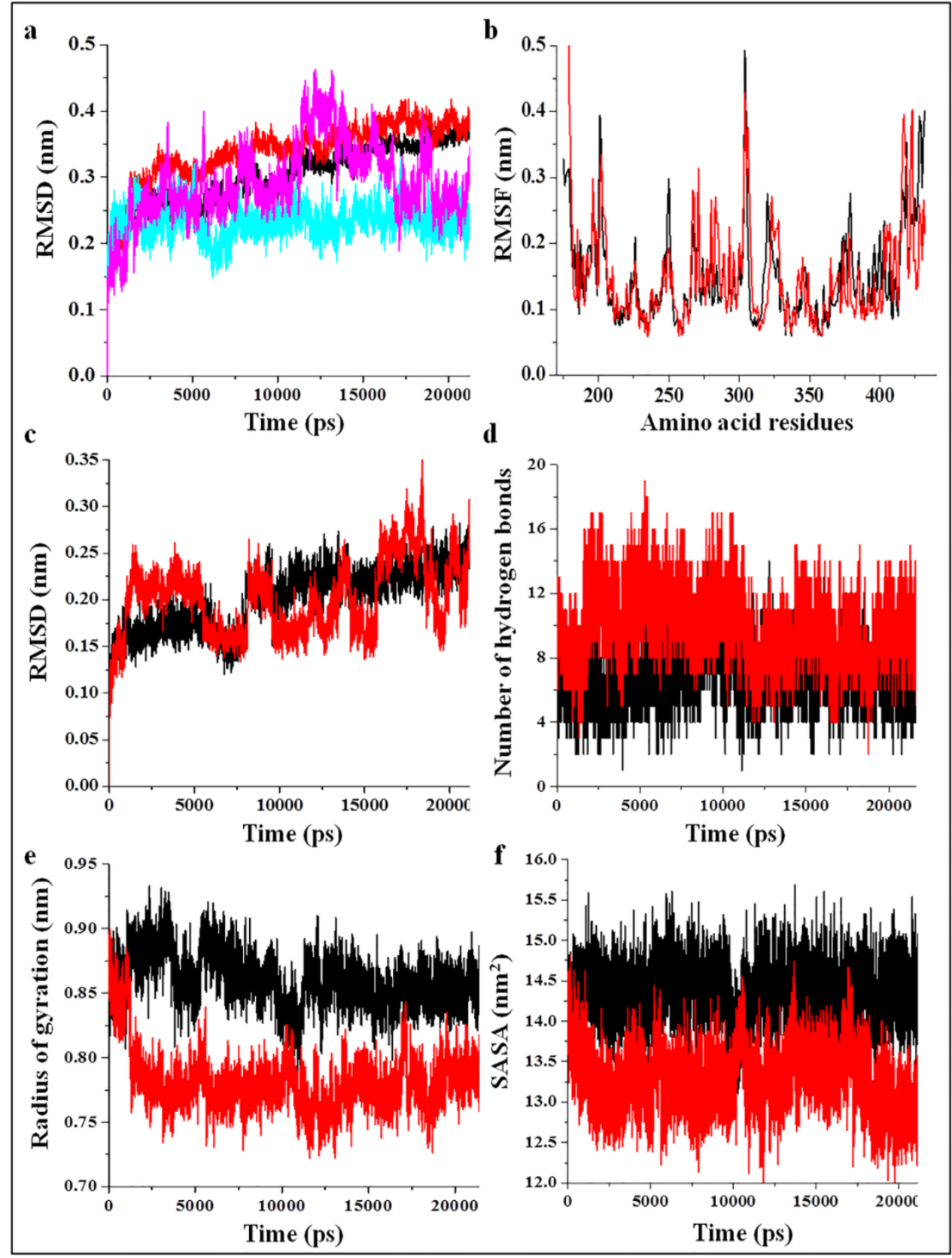

Fig. 2: Simulation-based evaluation of the binding interaction of WILD/K3C peptide with cyclin A. (a) RMSD plot of cyclin A protein (black and red), WILD (cyan) and K3C peptide (pink). (b) RMSF plot of cyclin A protein. (c) RMSD plot of substrate binding groove residues (Ser209, Met210, Ile213, Leu214, Asp216, Trp217, Glu220, Glu223, Glu224, Arg250, Leu253, Gln254, Tyr280, Ile281, Thr282, Asp283 and Asp284) of cyclin A. (d) Hydrogen bond plot of protein-peptide complex. (e) Rg plot of the WILD and K3C peptides. (f) SASA plot of the WILD and K3C peptides. Black and red lines correspond to WILD and $\mathrm{K} 3 \mathrm{C}$

Table 3: Cyclin A-WILD/K3C peptide interaction analysis during the simulation run

\begin{tabular}{ll}
\hline Mutants & Interacting amino acids \\
\hline WILD-10 ns & Met210, Thr207, Ile213, Trp217, Glu220, Glu224, Arg250, Leu253, Ile281, Thr282, Asp283, Gln407 \\
WILD-20 ns & Ser209, Ile213, Asp216, Trp217, Leu253, Glu224, Glu277, Ile281, Asp283, Asp284 \\
K3C-10 ns & Glu220, Met210, Ile213, Trp217, Glu224, Arg250, Leu253, Gln254, Ile281, Asp283, Asp284 \\
K3C-20 ns & Met210, Ile213, Trp217, Glu220, Glu224, Gly251, Leu253, Gln254, Tyr280, Ile281, Asp283 \\
\hline
\end{tabular}




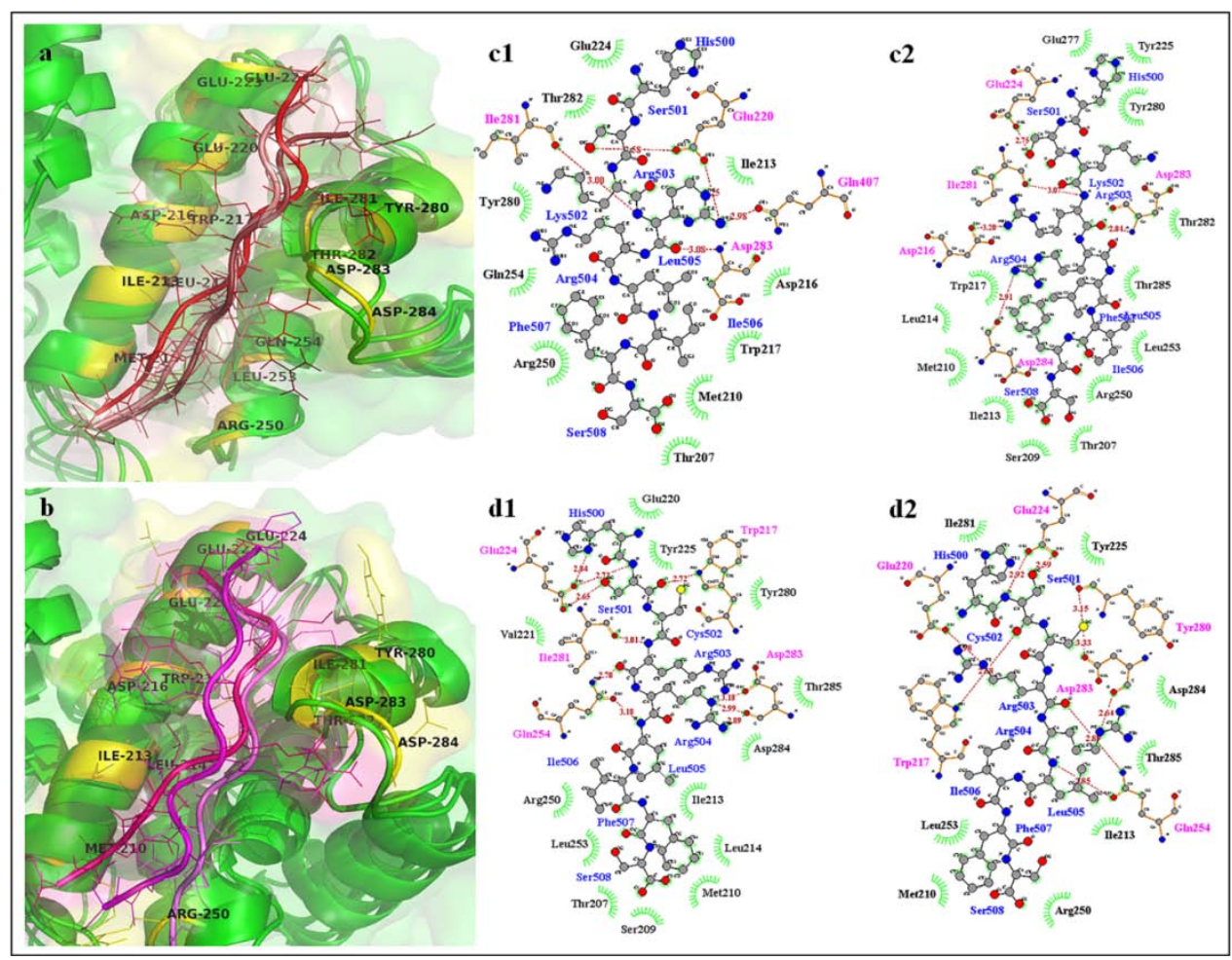

Fig. 3: Interaction analysis of cyclin A-WILD/K3C peptide complexes during simulation. (a) The structure of cyclin A (green) complexed with WILD peptide at $0 \mathrm{~ns}$ (red), $10 \mathrm{~ns}$ (salmon) and $20 \mathrm{~ns}$ (ruby). (b) Structure of cyclin A (green) complexed with K3C peptide at $0 \mathrm{~ns}$ (magenta), $10 \mathrm{~ns}$ (hot pink) and $20 \mathrm{~ns}$ (violet). (c) LigPlot plus based 2D interaction plot of the WILD peptide at $10 \mathrm{~ns}$ (c1) and $20 \mathrm{~ns}$ (c2).

(d) LigPlot plus based 2D interaction plot of the WILD peptide at $10 \mathrm{~ns}$ (d1) and $20 \mathrm{~ns}$ (d2). Amino acid His500-Ser508 of WILD/K3C peptide in the LigPlot plus 2D representation corresponds to His1-Ser9 (in reference to other sections of the manuscript)

Table 4: Per-residue interaction analysis of Cyclin A-WILD/K3C peptide complex during the simulation run

\begin{tabular}{lll}
\hline Cyclin A/WILD peptide & & \\
\hline Peptide residues & $\mathbf{1 0}$ ns & $\mathbf{2 0}$ ns \\
His1 & Glu224, Ile281 & Glu277 \\
Ser2 & Glu220 & Glu224 \\
Lys3 & Ile281 & Ile281, Asp283 \\
Arg4 & Trp217, Glu220, Ile281, Thr282, Asp283, Gln407 & Asp216, Ile281, Asp283 \\
Arg5 & Asp283 & Asp283, Asp284 \\
Leu6 & Ile213, Trp217 & Trp217 \\
Ile7 & - & - \\
Phe8 & Met210, Arg250, Leu253 & Ile213, Leu253 \\
Ser9 & Thr207 & Ser209 \\
Cyclin A/K3C peptide & & \\
His1 & Glu220, Glu224 & Glu220, Glu224 \\
Ser2 & Trp217, Glu224 & Trp217, Glu224 \\
Cys3 & Tyr280, Ile281, & Tyr280, Ile281, Asp283 \\
Arg4 & Trp217, Gln254, Ile281 & Trp217, Glu220, Gln254, Ile281, Asp283 \\
Arg5 & Asp283, Asp284 & Gln254, Asp283 \\
Leu6 & Ile213, Trp217, Gln254 & Gln254, Ile213 \\
Ile7 & - & - \\
Phe8 & Met210, Arg250, Leu253 & Gly251, Met210, Arg250, Leu253 \\
Ser9 & Met210 & - \\
\hline
\end{tabular}

Further, MM/PBSA analysis showed a significantly higher binding affinity of the K3C peptide for cyclin A in comparison to WILD. The binding energy of WILD and K3C peptide was-464.999 \pm 60.379 and$593.947 \pm 73.919 \mathrm{~kJ} / \mathrm{mol}$ respectively, at the end of the analysis. Moreover, the binding energy of K3C was more negative than WILD throughout the simulation run. The energy decomposition analysis revealed that the replacement of lysine by cysteine at the third position resulted in a decrease of polar solvation energy thereby increasing the total negative binding energy of the interaction (table 5). In addition, an insignificant decrease in van der Wall energy, electrostatic energy, and SASA energy was also observed. Per- residue energy decomposition energy also showed that most of the substrate binding site residues including Ser209, Met210, Ile213, Leu214, Asp216, Trp217, Glu220, Glu224, Arg250, and Leu253 had a greater contribution in the $\mathrm{K} 3 \mathrm{C}$ peptide (table 6). Here, it is important to mention that Glu223, Tyr280, Ile281, Thr282, Asp283, and Asp284 had a lower energy contribution for K3C than WILD type. This could possibly be due to the initial rolling of the $\mathrm{N}$ terminal and the absence of Lys3 residue in the $\mathrm{K} 3 \mathrm{C}$ mutant peptide. These results when taken together clearly suggest a higher affinity of K3C mutant over WILD type peptide and thus, it could potential application in cancer therapy. 
Table 5: Energy decomposition analysis of Cyclin A-WILD/K3C peptide complexes

\begin{tabular}{|c|c|c|c|c|c|}
\hline \multirow[t]{2}{*}{ Peptides } & \multicolumn{5}{|l|}{ Energy (kJ/mol) } \\
\hline & van der Waal energy & Electrostatic energy & Polar solvation energy & SASA energy & Binding energy \\
\hline WILD & $-236.402 \pm 20.502$ & $-2929.631 \pm 186.171$ & $2732.518 \pm 150.040$ & $-31.484 \pm 2.257$ & $-464.999 \pm 60.379$ \\
\hline $\mathrm{K} 3 \mathrm{C}$ & $-212.921 \pm 20.182$ & $-2711.249 \pm 217.242$ & $2360.062 \pm 151.357$ & $-29.840 \pm 1.912$ & $-593.947 \pm 73.919$ \\
\hline
\end{tabular}

Number of experiments, $\mathrm{n}=3$; data represented as mean $\pm \mathrm{SD}$

Table 6: Per-residue energy decomposition analysis of Cyclin A-WILD/K3C peptide complexes

\begin{tabular}{lll}
\hline Amino acid residues & \multicolumn{2}{l}{ Per residue binding energy (kJ/mol) } \\
\cline { 2 - 3 } & WILD & K3C \\
\hline Ser209 & $-1.825 \pm 0.0721$ & $-14.169 \pm 0.4936$ \\
Met210 & $-5.687 \pm 0.1235$ & $-11.068 \pm 0.1931$ \\
Ile213 & $-8.511 \pm 0.1464$ & $-16.489 \pm 0.1885$ \\
Leu214 & $-4.761 \pm 0.0557$ & $-8.432 \pm 0.0731$ \\
Asp216 & $-20.340 \pm 0.3495$ & $-53.983 \pm 0.5318$ \\
Trp217 & $-11.481 \pm 0.1533$ & $-14.930 \pm 0.2116$ \\
Glu220 & $-31.491 \pm 0.5294$ & $-66.021 \pm 0.8461$ \\
Glu223 & $-20.394 \pm 0.2369$ & $-16.704 \pm 0.2712$ \\
Glu224 & $-49.874 \pm 0.6252$ & $-53.443 \pm 0.57$ \\
Arg250 & $-10.401 \pm 0.569$ & $-18.106 \pm 0.4331$ \\
Leu253 & $-1.929 \pm 0.0606$ & $-2.428 \pm 0.0796$ \\
Glu254 & $-9.947 \pm 0.1537$ & $-9.531 \pm 0.1736$ \\
Tyr280 & $-19.594 \pm 0.2994$ & $-5.927 \pm 0.2331$ \\
Ile281 & $-19.645 \pm 0.1586$ & $-14.558 \pm 0.1396$ \\
Thr282 & $-4.884 \pm 0.1467$ & $-4.385 \pm 0.1253$ \\
Asp283 & $-46.995 \pm 0.6785$ & $-26.798 \pm 0.3892$ \\
Asp284 & $-27.736 \pm 0.3462$ & $-13.372 \pm 0.1968$ \\
\hline
\end{tabular}

Number of experiments, $\mathrm{n}=3$; data represented as mean $\pm \mathrm{SD}$

\section{CONCLUSION}

In the present work, we applied docking and molecular dynamic simulation approach to screen novel cyclin A inhibitors derived from the p21 peptide for an effective cancer therapy. Our analysis establishes that $\mathrm{K} 3 \mathrm{C}$ mutant peptide could bind at with cyclin A with much higher affinity that WILD peptide. Thus, it could prove to be a potential candidate as an anticancer agent. This is a preliminary study and further in depth in vitro and in vivo validations are required to validate this hypothesis.

\section{AUTHORS CONTRIBUTIONS}

TA and TKM developed the idea of the present work. TA, NA, HPR, and $\mathrm{SB}$ carried out the experimentation and analysis of the obtained results. TA, NA, and HBA together wrote the manuscript. HBA provided access to the computational facility present at Advanced Medical and Dental Institute, Universiti Sains Malaysia, Malaysia. TA, TKM, and HBA constantly reviewed the work and the manuscript.

\section{CONFLICTS OF INTERESTS}

There are no conflicts of interest

\section{REFERENCES}

1. Agarwal T, Annamalai N, Khursheed A, Maiti TK, Arsad HB, Siddiqui MH. Molecular docking and dynamic simulation evaluation of rohinitib-cantharidin based novel HSF1 inhibitors for cancer therapy. J Mol Graph Model 2015;61:141-9.

2. Chidambaram M, Manavalan R, Kathiresan K. Nanotherapeutics to overcome conventional cancer chemotherapy limitations. Int J Pharm Pharm Sci 2011;14:67-77.

3. Akhtar MJ, Ahamed M, Alhadlaq HA, Alrokayan SA, Kumar S. Targeted anticancer therapy: overexpressed receptors and nanotechnology. Clinica Chimica Acta 2014;436:78-92.

4. Lodish H, Berk A, Zipursky SL, Matsudaira P, Baltimore D, Darnell J. DNA damage and repair and their role in carcinogenesis; 2000.

5. Painter R. DNA damage and repair in eukaryotic cells. Genetics 1974;78:139-48.

6. Oren M. Regulation of the p53 tumor suppressor protein. J Biol Chem 1999;274:36031-4.

7. Goodsell DS. The molecular perspective: p53 tumor suppressor. Oncologist 1999;4:138-9.
8. Behera B, Mukherjee D, Agarwal T, Das J, Ghosh SK, Maiti TK. Cell penetrating peptides from agglutinin protein of Abrus precatorius facilitate the uptake of Imatinib mesylate. Colloids Surf B 2016;140:169-75.

9. Borghouts C, Kunz C, Groner B. Current strategies for the development of peptide-based anti-cancer therapeutics. J Pept Sci 2005;11:713-26.

10. Zheleva D, McInnes C, Gavine AL, Zhelev N, Fischer P, Lane D. Highly potent p21WAFarived peptide inhibitors of CDK-mediated pRb phosphorylation: delineation and structural insight into their interactions with cyclin a. J Pept Sci 2002;60:257-70.

11. R Pincus M, Fenelus M, Sarafraz Yazdi E, Adler V, Bowne W, Michl J. Anti-cancer peptides from ras-p21 and p53 proteins. Curr Pharm Des 2011;17:2677-98.

12. Fischer PM, Lane DP. Inhibitors of cyclin-dependent kinases as anti-cancer therapeutics. Curr Med Chem 2000; 7:1213-45.

13. Russo AA, Jeffrey PD, Patten AK, Massague J, Pavletich NP. Crystal structure of the p27Kip1 cyclin-dependent-kinase inhibitor bound to the cyclin A-Cdk2 complex. Nature 1996;382:325-31.

14. Gulbis JM, Kelman Z, Hurwitz J, O'Donnell M, Kuriyan J. Structure of the C-terminal region of p21 WAF1/CIP1 complexed with human PCNA. Cell 1996;87:297-306.

15. Tyagi A, Kapoor P, Kumar R, Chaudhary K, Gautam A, Raghava $\mathrm{G}$. In silico models for designing and discovering novel anticancer peptides. Sci Rep 2013;3:2984.

16. Kozakov D, Brenke R, Comeau SR, Vajda S. PIPER: an FFT-based protein docking program with pairwise potentials. Proteins: Struct Funct Bioinf 2006;65:392-406.

17. Comeau SR, Gatchell DW, Vajda S, Camacho CJ. ClusPro: an automated docking and discrimination method for the prediction of protein complexes. Bioinformatics 2004;20:45-50.

18. Anand Thiyagaraj JSS, Waheeta Sopper. Effect of toll-like receptor inhibitor imiquimod on IL1R1 interaction with IL1Ra and its SNP variant-an in silico approach. Int J Pharm Pharm Sci 2016;8:109-12.

19. Rakesh KR, Pandit DG, Tapan K Mukherjee. Identification of potential salmonella typhi beta-lactamase tem 1 inhibitors using peptidomimetics, virtual screening, and molecular dynamics simulations. Int J Pharm Pharm Sci 2018;10:91-6. 